\title{
Detecting Undernutrition on Hospital Admission - Screening Tool Versus WHO Criteria
}

\author{
Zrinka Matak $^{1, ~ *, ~ D u s k a ~ T j e s i c-D r i n k o v i c ~}{ }^{1,2}$, Lana Omerza ${ }^{2}$, Irena Senecic-Cala ${ }^{1,2}$, \\ Jurica Vukovic $^{1,2}$, Dorian Tjesic-Drinkovic ${ }^{1,2}$ \\ ${ }^{1}$ Zagreb School of Medicine, Zagreb, Croatia \\ ${ }^{2}$ Department of Pediatrics, University Hospital Centre Zagreb, Zagreb, Croatia
}

Email address:

zrinka.matak@gmail.com (Z. Matak),duska.td@gmail.com (D.Tjesic-Drinkovic), lanamadercic@yahoo.com (L. Omerza), isenecic@yahoo.com (I. Senecic-Cala),juricav1961@yahoo.com (J. Vukovic),dorian.td@gmail.com (D. Tjesic-Drinkovic) ${ }^{*}$ Corresponding author

\section{To cite this article:}

Zrinka Matak, Duška Tjesic-Drinkovic, Lana Omerza, Irena Senecic-Cala, Jurica Vukovic, Dorian Tjesic-Drinkovic. Detecting Undernutrition on Hospital Admission - Screening Tool Versus WHO Criteria. Clinical Medicine Research. Vol. 6, No. 3, 2017, pp. 74-79. doi: 10.11648/j.cmr.20170603.13

Received: January 27, 2017; Accepted: March 14, 2017; Published: April 10, 2017

\begin{abstract}
To prevent the development of malnutrition in hospitalized children, it is important to develope an early identification of nutritional depletion, ideally at the time of admission to the hospital. In 2009 Hulst et al. proposed new guidelines for assessing the nutritional status of hospitalized children called STRONGkids questionnaire (Screening Tool Risk on Nutritional Status and Growth). This study was designed to describe the current prevalence of malnutrition on admission to a pediatric gastroenterology hospital unit and to compare the value and feasibility of STRONGkids scoring system versus anthropometric World Health Organization (WHO) criteria in identifying children at risk of developing malnutrition during hospital stay. The prospective observational study involved 124 children hospitalized at the Department of Pediatrics, University Hospital Center Zagreb. Nutritional status and risk for malnutrition were estimated by STRONGkids questionnaire and anthropometric measurements of subjects. Statistical analysis was performed using the computer program STATISTICS 10, StatSoft. Inc. 1984-2011; using descriptive statistics, Fisher's exact (FE) test and non-parametric Kruskal-Wallis (KW) test. Total malnutrition was observed in $18.5 \%$ of patients. Larger number of children at risk for malnutrition were identified by STRONGkids questionnaire than by anthropometric measurements (STRONGkids questionnaire: $75.8 \%$; anthropometric measures: $40.3 \%$ ). Patients that lost weight during hospitalization (33.1\%) were further analyzed: $8 / 41$ were not detected to be at risk by either method, 11/41 were identified by STRONGkids and anthropometry, and 22/41 were detected only by STRONGkids (Fisher's exact test $\mathrm{p}=0,08$ ). This study justifies the inclusion of the STRONGkids questionnaire in the initial evaluation of children on admission to the hospital, in order to recognize those who need special nutritional support and thus prevent the development of malnutrition during hospitalization.
\end{abstract}

Keywords: Nutritional Status, Hospitalized Children, Malnutrition, STRONGkids, Screening Tool, Anthropometry

\section{Introduction}

Malnutrition is as a state of nutrition in which deficiency, excess or imbalance of energy, protein, and other nutrients causes measurable adverse effects on the body and upon growth that may increase morbidity or mortality [1]. Children who are admitted to the hospital are at risk of developing malnutrition, especially children with an underlying disease [2]. Hospital malnutrition and its adverse consequences were already described in various countries around the world. Lowest rates have been reported in developed countries such as Germany (6.1\%) and France (21\%); higher rates in developing countries such as Romania (37\%) and Turkey (31.8\%); and the highest rates in undeveloped countries such as Iran (67.07\%) [3]. Given the repercussions of malnutrition in patient 
morbidity-mortality, prolonged hospital stay and healthcare costs, in order to prevent malnutrition and especially hospital-acquired malnutrition, nutritional screening measures must be placed as a first step in an integrated nutritional care plan for patients during hospitalization. Due to the lack of a simple and valid nutritional screening tool routine nutritional screening is rarely carried out in pediatric patients. Although numerous simple screening tools have been developed none has been accepted as a universal tool [4-5]. In 2009 Hulst et al. designed new guidelines for assessing the nutritional status of hospitalized children called STRONGkids questionnaire (Screening Tool Risk on Nutritional Status and Growth) [6]. The aim of this study is to describe the current prevalence of malnutrition on admission to a pediatric gastroenterology hospital unit and compare the value and feasibility of STRONGkids scoring system versus anthropometric World Health Organization (WHO) criteria in identifying children at risk of developing malnutrition during hospital stay.

\section{Methods}

A prospective observational study was undertaken at the Division of Pediatric Gastroenterology, University Hospital Centre Zagreb in the period January-April in 2013 and in the same period in 2014. Children were evaluated on admission. Inclusion criteria were child's age above 1 month, parent/guardian consent to participate and availability within the first $24 \mathrm{~h}$ for the interview and expected hospital stay of minimum 48 hours. The nutritional status of hospitalized children has been evaluated by one, always the same investigator in two ways: using nutritional risk screening tool, called STRONGkids and using anthropometric WHO criteria. The parents and, when possible, the patients were interviewed using STRONGkids questionnaire (Table 1) within $48 \mathrm{~h}$ of hospital admission. The points awarded by STRONGkids questionnaire were totaled and then used to classify the patient's nutritional status called STRONGkids score. A total score of 0 points classified the patients as low/not at risk; $1-3$ classified the patients as at moderate risk; and 4-5 classified patients as high risk.

Table 1. STRONGkids (Screening Tool Risk on Nutritional Status and Growth) questionnaire.

\begin{tabular}{|c|c|c|c|}
\hline \multirow{2}{*}{$\begin{array}{l}\text { - Hospital No } \\
\text { - Name } \\
\text { - Surname } \\
\text { - Sex } \\
\text { - Age } \\
\text { - Diagnosis } \\
\end{array}$} & \multicolumn{3}{|l|}{$\begin{array}{l}\text { - Date of hospital admission } \\
\text { - Height/length } \\
\text { - Weight } \\
\end{array}$} \\
\hline & \multicolumn{3}{|l|}{$\begin{array}{l}\text { - Date of hospital discharge } \\
\text { - Weight }\end{array}$} \\
\hline \multicolumn{4}{|l|}{ STRONGkids } \\
\hline (1) Subjective clinical assessment & $\begin{array}{l}\text { - Is the patient in a poor nutritional status judged by subjective clinical assessment } \\
\text { (diminished subcutaneous fat and/or muscle mass and/or hollow face)? }\end{array}$ & $\begin{array}{l}\text { NO } \\
\text { YES }\end{array}$ & $\begin{array}{l}0 \\
1\end{array}$ \\
\hline (2) High risk disease & - Is there an underlying illness with a risk of malnutrition or expected major surgery & $\begin{array}{l}\text { NO } \\
\text { YES }\end{array}$ & $\begin{array}{l}0 \\
2\end{array}$ \\
\hline (3) Nutritional intake and losses & $\begin{array}{l}\text { - Are one of the following items present? } \\
\text { Excessive diarrhoea ( } 5 \text { per day) and/or vomiting ( }>3 \text { times/day) the last few days? } \\
\text { Reduced food intake during the last few days before admission (not including fasting for an } \\
\text { elective procedure or surgery)? } \\
\text { Pre-existing dietetically advised nutritional intervention? }\end{array}$ & NO & 1 \\
\hline Calculate total score (total of items 1-4) & Total nutritional STRONGkids score & & \\
\hline
\end{tabular}

Patients were measured using standard anthropometric equipment immediately upon admission to the hospital, and also at discharge. Anthropometric data was compared with WHO international reference standards and expressed as $z$-scores for body mass index-for-age (BMI-for-age) to indicate acute malnutrition and as $z$-scores for height-forage (HFA) to indicate chronic malnutrition [7-8]. A $z$ score of more than 2 standard deviation (SD) below the mean was used to define children as malnourished and a $z$ score between -1 SD and -2 SD was used to define children as moderately undernourished or at risk of becoming severely undernourished. Statistical analysis was performed using the computer program STATISTICS 10, StatSoft. Inc. 1984-2011; using descriptive statistics,
Fisher's exact (FE) test and non-parametric Kruskal-Wallis (KW) test. Statistical significance was set at $p<0.05$. All aspects of the present study were approved by the University Hospital Center Zagreb Ethical Committee and by the Ethical Committee of the Zagreb School of Medicine.

\section{Results}

In the observed period, 143 children were admitted, and 124 fulfilling the inclusion criteria patients (67 male and 57 female) were included in the study. The median age was 10.35 years (range 0.08-17.91 years). Baseline characteristics are shown in Table 2. 
Table 2. Characteristics of patients; total and divided in three STRONGkids (Screening Tool Risk on Nutritional Status and Growth) nutritional risk groups.

\begin{tabular}{|c|c|c|c|c|}
\hline & \multicolumn{4}{|c|}{ Risk for malnutrition according to STRONGkids } \\
\hline & Total $(\mathrm{N}=124)$ & Low risk $(\mathrm{N}=\mathbf{3 0})$ & Moderate risk ( $\mathbf{N}=\mathbf{8 0}$ ) & High risk $(\mathrm{N}=14)$ \\
\hline BMI-for-age $z$-score (median) $^{*}$ & -0.46 & 0.92 & -0.56 & -2.67 \\
\hline Height-for-age $z$-score (median) & 0.25 & 0.96 & 0.24 & -0.87 \\
\hline Acute malnutrition, $\mathrm{N}(\%)$ & $12(9.7)$ & $0(0)$ & $3(2.4)$ & $9(7.3)$ \\
\hline Chronic malnutrition, $\mathrm{N}(\%)$ & $13(10.5)$ & $5(4)$ & $5(4)$ & $3(2.4)$ \\
\hline Malnutrition, $\mathrm{N}(\%)$ & $23(18.5)$ & $5(16.7)$ & $7(8.6)$ & $11(78.6)$ \\
\hline Age (median, years) ${ }^{* *}$ & 10.35 & 12.9 & 10.36 & 0.4 \\
\hline Underlying disease, $\mathrm{N}$ & 51 & 0 & 37 & 14 \\
\hline $\begin{array}{l}\text { Length of hospital stay (median, } \\
\text { days) }\end{array}$ & 6.5 & 4 & 7 & 14 \\
\hline
\end{tabular}

Significant difference between low, moderate and high risk group:

* Kruskal-Wallis $(\mathrm{KW})$ test $=62.69 ; \mathrm{p}<0.0001$

** Kruskal-Wallis $(\mathrm{KW})$ test $=21.29 ; \mathrm{p}=0.0001$

*** Kruskal-Wallis $(\mathrm{KW})$ test $=19.05 ; \mathrm{p}<0.0001$

Acute malnutrition was observed in $9.7 \%$ of patients, and chronic malnutrition in $10.5 \%$ of patients. Total malnutrition (defined by the presence of acute and /or chronic malnutrition) was observed in $18.5 \%$ of patients. The points awarded to each child by STRONGkids questionnaire were totaled as follows: 30 children $(24.2 \%)$ had a total STRONGkids score 0 points; 27 (21.8\%) had 1; 34 (27.4\%) had 2; $19(15.3 \%)$ had 3; $9(7.3 \%)$ had 4 and 5 (4\%) patients had 5 points. STRONGkids divided the children into three risk groups: 30 children (24.2\%) were scored at low risk, 80 $(64.5 \%)$ at moderate risk, and $14(11.3 \%)$ children were considered to be at high nutritional risk (Table 2). A statistically significant correlation between the total STRONGkids score and BMI-for-age was observed (KW test $=69.1 ; p<0.0001$; Fig. 1 ), as well as a significant difference among BMI-for-age of patients in different risk groups defined by STRONGkids (KW 62.7; p<0.0001; Fig. 2).

$K W$ test $=69.1 ; p<0.0001$

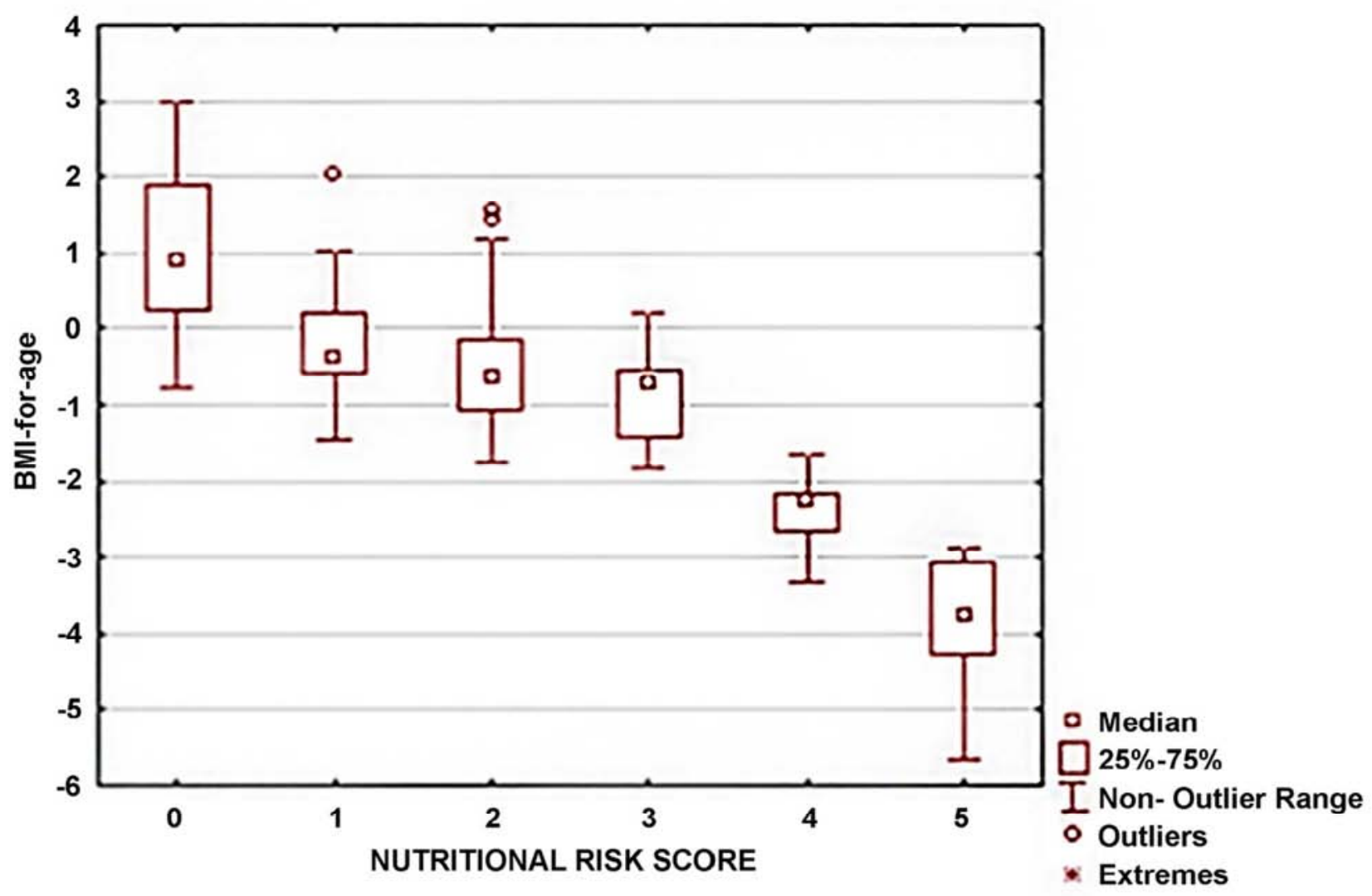

$\mathrm{KW}=$ Kruskal-Wallis test.

Figure 1. Correlation of nutritional risk scores (STRONGkids) and body mass index-for-age (BMI-for-age). The data are presented as nutritional risk score (scale 0-5) assessed by STRONGkids tool and median of z-score for BMI-for age. 
$\mathrm{KW}$ test $=62.7 ; p<0.0001$

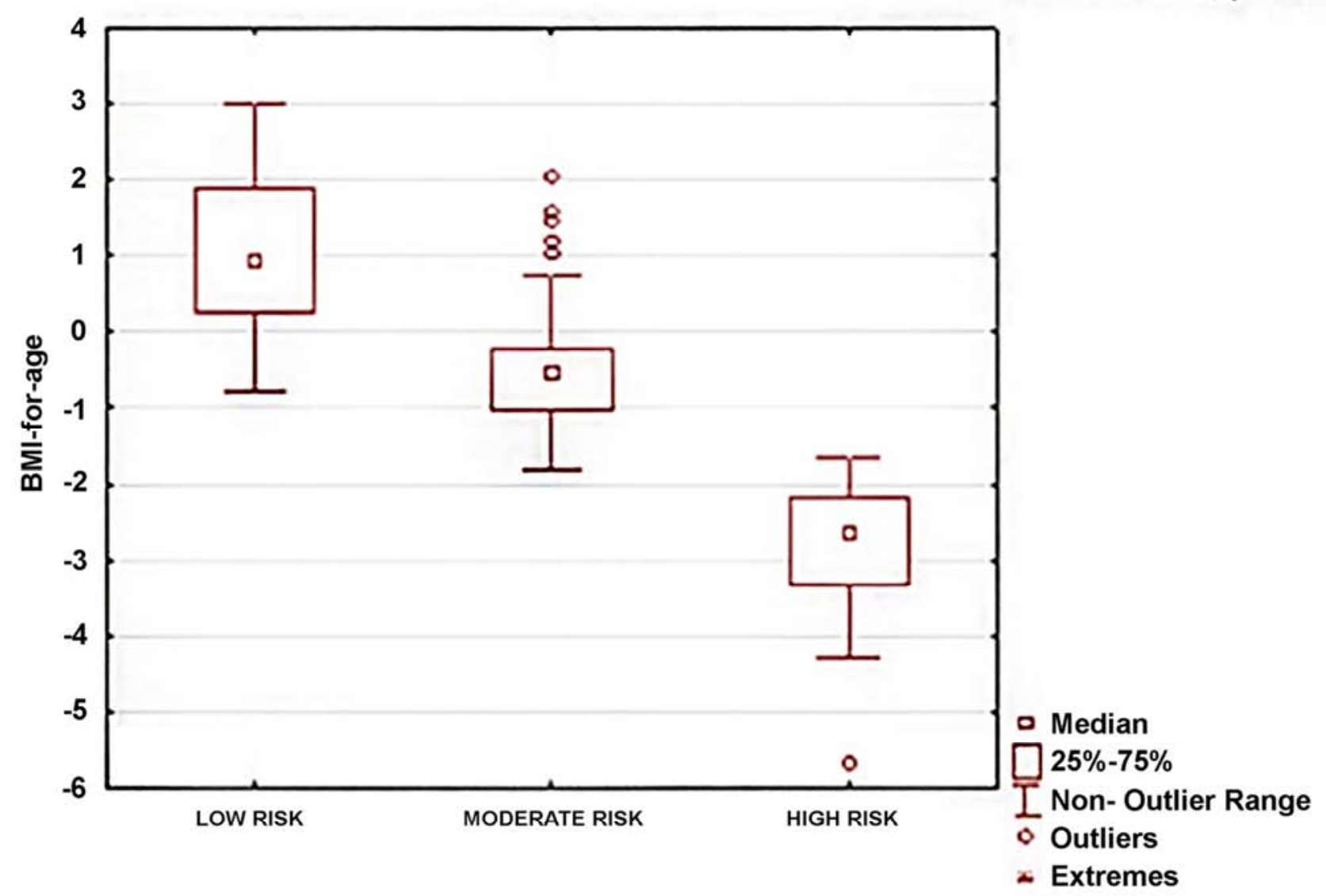

$\mathrm{KW}=$ Kruskal-Wallis test

Figure 2. Median body mass index-for-age (BMI-for-age) in the low, medium and high risk group for malnutrition based on the STRONGkids scoring system.

Furthermore, the age of children differed significantly among different risk groups, the younger contributing mostly to the high risk group $(\mathrm{p}=0.0001$, Table 2$)$. No correlation between HFA $z$-scores and STRONGkids risk categories was present $(\mathrm{p}=0.19)$. The percentage of children suffering from an underlying disease increased with each nutritional risk level: $0 \%$ in the low-risk group, $46.3 \%$ in moderate-risk group and $100 \%$ in high-risk group. Median duration of hospitalization was 6.5 days (range 2-64 days). Children with higher risk for malnutrition stayed longer in hospital (KW test $=19.05 ; \mathrm{p}<0.0001)$. Patients that lost weight during hospitalization $(33.1 \%)$ were further analyzed: $8 / 41$ were not detected to be at risk by either method, 11/41 were identified by STRONGkids and anthropometry, and $22 / 41$ were detected only by STRONGkids (Fisher's exact test $\mathrm{p}=0,08$ ).

\section{Discussion}

In this study, the most established way to describe malnutrition (WHO definition with $z$-cores) was used [7-8]. The strength of the present study is that one researcher facilitated and conducted all anthropometric measures, which eliminated interobserver measurement variability. Age- and gender specific $z$-scores calculated using WHO reference data confirmed the serious issue of malnutrition among children hospitalized due to gastroenterological diseases. The detected prevalence of malnutrition on hospital admission is $18.5 \%$ and is in accordance with figures form Europe [3]. This high prevalence, almost $1 / 5$ admitted children, can be partially explained by the specificity of patients. The most prevalent diagnoses were inflammatory bowel disease and chronic liver disease, conditions that directly influence nutritional status [9]. All malnourished children undoubtedly deserve special nutritional support during hospital stay. It is reasonable to assume that optimizing nutrition so that the child is less malnourished (i.e., mild instead of severe) would result in more favorable outcomes. Using $z$-scores is a good method of following these patients, especially in those severely malnourished, because it enables measuring i.e. objectivizing improvement upon nutritional interventions in the subgroup of children with extreme values that would otherwise be considered just "under the curve".

Hospitalization itself is a risk for weight lost [10], so borderline nourished patients can be considered to be at danger of developing undernutrition. In attempt to maximize the power of anthropometric measures in identifying all children that would need nutritional supervision, borderline nourished patients on admission were added to malnourished group, mounting to $50 / 124$ patients $(40.3 \%)$. These patients can be considered as those at risk. However, the 
STRONGkids screening tool recognized considerably more children prone to developing malnutrition; $75.8 \%$.

This difference rises the possibility that STRONGkids overestimates the nutritional risk. In order to indirectly check this possibility, we analyzed the subset of 41/124 children that actually lost weight during hospitalization. Twice more patients (22/41 versus 11/41) were (correctly) identified to be at risk using STRONGkids in comparison to the WHO criteria. Even though the statistical significance was not confirmed (Fisher's exact test $\mathrm{p}=0,08$ ), this implicates STRONGkids' strength and superiority in identifying children who are at risk of weight loss during hospitalization and emphasizes the importance of taking into account other risk factors in addition to anthropometric measures. In fact, illness related malnutrition has complex etiology, and STRONGkids is tailored to address some of these aspects such as eating habits, nutritional losses and the disease itself together with the appearance of the patient. It is worth mentioning that even with STRONGkids tool 8/41 patients that lost weight were not recognized as being at risk. This leaves the possibility to look for some other important risk factor and maybe use it to modify STRONGkids. Since the data on malnutrition of children admitted to hospital are strictly dependent on the methods used for its recognition [11], it is important to have a simple and reliable tool and try to improve the existing ones.

Taking in consideration that the STRONGkids score depends on no actual body measurements, we compared the anthropometric data with data obtained by STRONGkids to validate its reliability for recognizing malnutrition risk. We found a significant relationship of the current nutritional status with STRONGkids (BMI-for-age $z$-score was negatively correlated with STRONGkids risk categories). We also found a significant relationship between having a "high risk" score and younger age of the children. These results are in accordance not only with findings of the original publication of the STRONGkids in Dutch population but also with another recent study that tested the use of the STRONGkids in Italian hospitalized children $[6,12]$. This altogether confirms the validity of the STRONGkids questionnaire in different countries, as well as in Croatia.

The newest prospective European multi-center cohort study proved that malnutrition is associated with longer length of hospital stay [13]. Our results confirmed that children with lower BMI-for-age stayed longer in hospital, and we found a significant relationship between having a "high risk" score and prolonged hospital stay. This is in concordance to previous studies concerning STRONGkids $[6,14-15]$.

Our study demonstrated that STRONGkids screening tool is easy to use and convenient (one examiner is sufficient, no time consumption and no possibility of measurement errors), it gives reliable information on nutritional status (correlation of results with BMI-for-age) and is superior to the classical criteria of $\mathrm{WHO}$ in identifying hospitalized children at nutritional risk.

\section{Conclusions}

This study showed that malnutrition in children with gastrointestinal diseases is present among children admitted to a Croatian hospital in a percentage similar to other European countries [9]. Results from STRONGkids screening tool correlated well with BMI-for-age, the anthropometric World Health Organization (WHO) criteria for malnutrition. However, STRONGkids questionnaire was superior to anthropometric WHO criteria in detecting patients at risk for malnutrition and in identifying those children who actually lost weight during hospitalization. This study demonstrated the STRONGkids screening tool credibility and convenience. We recommend engaging STRONGkids questionnaire in the initial evaluation of children on admission to the hospital, in order to timely identify individuals in need of specially planned nutritional support. The appreciation of nutritional teams and the importance of malnutrition need to be strengthened in pediatric hospitals.

\section{Ethical Approval}

All procedures performed in studies involving human participants were in accordance with the ethical standards of the institutional and/or national research committee and with the 1964 Helsinki declaration and its later amendments or comparable ethical standards. All aspects of the present study were approved by the University Hospital Center Zagreb Ethical Committee and by the Ethical Committee of the Zagreb School of Medicine.

\section{References}

[1] Lochs H, Allison SP, Meier R, et al. Introductory to the ESPEN Guidelines on Enteral Nutrition: Terminology, definitions and general topics. Clin Nutr. 2006; 25:180-186.

[2] Mosby TT. Almost a fifth of children admitted to Dutch hospitals have acute or chronic malnutrition; risk factors include underlying disease and non-white ethnicity. Evid Based Nurs. 2010; 13:81-82.

[3] Kapçı N, Akçam M, Koca T, Dereci S, Kapcı M. The nutritional status of hospitalized children: Has this subject been overlooked? Turk J Gastroenterol. 2015; 26:351-355.

[4] Hartman C, Shamir R, Hecht C, Koletzko B. Malnutrition screening tools for hospitalized Children. Curr Opin Clin Nutr Metab Care. 2012; 15(3):303-309.

[5] Aurangzeb B, Whitten KE, Harrison B, et al. Prevalence of malnutrition and risk of under-nutrition in hospitalized children. Clin Nutr. 2012; 31(1):35-40.

[6] Hulst JM, Zwart H, Hop WC, Joosten KFM. Dutch national survey to test the STRONGkids nutritional risk screening tool in hospitalized children. Clin Nutr. 2010; 29:106-111.

[7] WHO Multicentre Growth Reference Study Group. WHO Child Growth Standards based on length/height, weight and age. Acta Pediatr. 2006; 450:1-101. 
[8] World Health Organization. Child growth standards: WHO Anthro (version 3.2.2, January 2011) and macros countries [Internet]. World Health Organization. 2011. Available at: www.who.int/childgrowth/software/en/. Accessed 02 Mai 2013.

[9] Koen FM, Joosten MD, Jessie M, Hulst MD. Malnutrition in pediatric hospital patients: Current issues. Nutrition. 2011; 27:133-137.

[10] Hwang EH, Park JH, Chun P, Lee YJ. Prevalence and Risk Factors for the Weight Loss during Hospitalization in Children: A Single Korean Children's Hospital Experience. Pediatr Gastroenterol Hepatol Nutr. 2016; 19(4):269-275.

[11] Joosten KFM, Hulst JM. Prevalence of malnutrition in pediatric hospital patients. Curr Opin Pediatr. 2008; 20:590 596.
[12] Spagnuolo MI, Liguoro I, Chiatto F, Mambretti D, Guarino A. Application of a score system to evaluate the risk of malnutrition in a multiple hospital setting. Ital $\mathrm{J}$ Pediatr.2013;39:81.

[13] Hecht C,Weber M, Grote V, et al. Disease associated malnutrition correlates with length of hospital stay in children. Clin Nutr. 2015; 34:53-59.

[14] Huysentruyt K, Alliet P, Muyshont L, et al. The STRONGkids nutritional screening tool in hospitalized children: A validation study. Nutrition. 2013; 29:1356-1361.

[15] Moeeni V, Walls T, Day AS. Assessment of nutritional status and nutritional risk in hospitalized Iranian children. Acta Paediatr. 2012; 101:446-451. 